\title{
REMARK ON COMMUTATIVE APPROXIMATE IDENTITIES ON HOMOGENEOUS GROUPS
}

\author{
JACEK DZIUBAŃSKI
}

(Communicated by J. Marshall Ash)

\begin{abstract}
We give, using the functional calculus of Hulanicki [4], a construction of a commutative approximate identity on every homogeneous group.
\end{abstract}

In [2] Folland and Stein asked, whether on every homogeneous group $\mathscr{N}$ there is a function $\phi$ in the Schwartz class $\mathscr{S}(\mathscr{N})$ with the following properties: $\int_{\mathscr{N}} \phi(x) d x=1, \phi_{t} * \phi_{s}=\phi_{s} * \phi_{t}$ where $\phi_{t}(x)=t^{-Q} \phi\left(\delta_{t^{-1}} x\right)$, and $Q$ is the homogeneous dimension of $\mathscr{N}$. The family $\left\{\phi_{t}\right\}$ is then called a commutative approximate identity and is used for characterizing Hardy spaces $H^{p}(\mathscr{N})$, cf. [2]. Folland and Stein [2] produced a commutative approximate identity in the case when $\mathcal{N}$ is graded. Glowacki showed in [3] that for every homogeneous group the densities of the stable semigroup of symmetric measures generated by the functional

$$
\langle P, f\rangle=\lim _{\varepsilon \rightarrow 0} \int_{|x|>\varepsilon} \frac{f(0)-f(x)}{|x|^{Q+1}} \Omega(x) d x,
$$

(where $\Omega$ is a nonzero nonnegative smooth away from the origin homogeneous of degree 0 symmetric function and $|\cdot|$ is a smooth homogeneous norm on $\mathscr{N}$ ) belong to $\mathscr{R}(\mathscr{N})$, cf. [2, p. 253]. The construction of a commutative approximate identity from such a semigroup of measures was presented in [2, pp. 258-260]. The purpose of this note is to give (using the functional calculus of Hulanicki [4]) an alternative construction of a commutative approximate identity from the distribution $P$. We prove that on every homogeneous group $\mathscr{N}$ the following theorem holds.

Theorem 1. If $F \in C_{c}(-1,1), F=1$ in a neighborhood of 0 , then there is a function $\phi \in \mathscr{S}(\mathscr{N})$ such that $\int_{0}^{\infty} F(\lambda) d E_{P}(\lambda) f=f * \phi$, where $E_{P}$ is the spectral resolution of the operator $f \mapsto P f=f * P$.

Corollary 1. Since $\int_{\mathscr{N}} \phi(x) d x=F(0)$ and $\int_{0}^{\infty} F(t \lambda) d E_{P}(\lambda) f=f * \phi_{t}$, the family $\left\{\phi_{t}\right\}$ forms a commutative approximate identity.

Let $U=\{x \in \mathscr{N}:|x| \leq 1\}$ and $\tau(x)=\inf \left\{n: x \in U^{n}\right\}$. For every $\alpha \geq 0$ the function $w_{\alpha}(x)=(1+\tau(x))^{\alpha}$ is submultiplicative. Moreover, there are

Received by the editors August 28, 1990.

1991 Mathematics Subject Classification. Primary 43A80.

Key words and phrases. Homogeneous groups. 
constants $c, C, a, b>0$ such that $a<1,2<b$, and

$$
c \tau(x)^{a} \leq|x| \leq C \tau(x)^{b} \text { for }|x|>1,
$$

cf. [4, Lemma 1.1]. Denote by $M_{\alpha}$ the *algebra of Borel measures $\mu$ on $\mathscr{N}$ such that $\int \omega_{\alpha}(x) d|\mu|(x)<\infty$. If $A$ is a selfadjoint operator on $L^{2}(\mathscr{N})$, $E_{A}$ is its spectral resolution, and $m$ is a bounded function on $\mathbf{R}$, then denote by $m(A)$ the operator $\int_{\mathbf{R}} m(\lambda) d E_{A}(\lambda)$. If $A f=f * \psi$ then $m(\psi)$ is the abbreviation for $m(A)$. The following theorem has been proved in [4]

Theorem 2. Suppose that $\psi=\psi^{*} \in M_{\alpha} \cap L^{2}(\mathscr{N}), \alpha>\beta+\frac{b}{2} Q+2, m \in C_{c}^{k}(\mathbf{R})$ with $k>3\left(\beta+\frac{b}{2} Q+3\right), m(0)=0$, then there is a measure $\nu$ in $M_{\beta}$ such that $m(\psi) f=f * \nu$.

The theorem below, which has been actually proved in [1], plays here the fundamental role.

Theorem 3. For every natural $N>Q$, let $T_{t}^{(N)}$ be the semigroup of operators on $L^{2}(\mathscr{N})$ generated by $P^{N}$. Then $T_{t}^{(N)} f=f * q_{t}^{(N)}$, where $q_{t}^{(N)} \in C^{\infty}(\mathcal{N})$ and $\sup _{x \in \mathcal{N}}\left|\partial q_{t}^{(N)}(x)\right|(1+|x|)^{N+Q} \leq C(t, \partial, N)<\infty$ for every left-invariant differential operator $\partial$ on $\mathscr{N}$.

Proposition 1. If $m \in C_{c}^{\infty}(-1,1)$, then for every natural $N>\left(\beta+\frac{b}{2} Q+2\right) / a$ there is a unique function $\phi$ such that $m\left(P^{N}\right)=f * \phi$. Moreover, for every left-invariant differential operator $\partial$ on $\mathscr{N}, \sup _{x \in \mathcal{N}}|\partial \phi(x)| w_{\beta}(x)<\infty$.

Proof. Let $n(\lambda)=m(-\log \lambda) / \lambda$. Then $n \in C_{c}^{\infty}\left(e^{-1}, e\right)$. Moreover, $m\left(P^{N}\right) f=$ $T_{1}^{(N)} n\left(q_{1}^{(N)}\right) f$. By Theorems 2, 3 and (1), $n\left(q_{1}^{(N)}\right) f=f * \nu$ holds with $\nu \in M_{\beta}$. Using Theorem 3 one obtains that $\phi=\nu * q_{1}^{(N)}$ satisfies required conditions.

Proof of Theorem 1. In virtue of (1) it suffices to show that there is a $C^{\infty}$ function $\phi$ such that for every $\beta>0$ and every left-invariant differential operator $\partial$, one has $\int_{0}^{\infty} F(\lambda) d E_{P}(\lambda) f=f * \phi$ and $\sup _{x \in \mathcal{N}}|\partial \phi(x)| w_{\beta}(x)<\infty$. But for every natural $N$

$$
\int_{0}^{\infty} F(\lambda) d E_{P}(\lambda) f=\int_{0}^{\infty} F_{N}(\lambda) d E_{P^{N}}(\lambda) f,
$$

where $F_{N}(\lambda)=F\left(|\lambda|^{1 / N}\right)$. By the definition of $F, F_{N} \in C_{c}^{\infty}(-1,1)$. Using (2) and Proposition 1 with sufficiently large $N$, the required estimate follows.

\section{REFERENCES}

1. J. Dziubański, A remark on a Marcinkiewicz-Hörmander multiplier theorem for some nondifferential convolution operators, Colloq. Math. 58 (1989), 77-83.

2. G. B. Folland and E. M. Stein, Hardy spaces on homogeneous groups, Princeton Univ. Press, Princeton, NJ, 1982.

3. P. Glowacki, Stable semigroup of measures as commutative approximate identities on nongraded homogeneous groups, Invent. Math. 83 (1986), 557-582.

4. A. Hulanicki, A functional calculus for Rockland operators on nilpotent Lie groups, Studia Math. 78 (1984), 253-266.

Mathematical Institute, Wroclaw University, PL. GrunWaldzki 2/4, 50-384 Wroclaw, POLAND 